\title{
Identifying charge-exchange collision products within the ion-energy distribution of electrostatically accelerated plasmas
}

\author{
Lyon B. King a) and Alec D. Gallimore ${ }^{\text {b) }}$ \\ Plasmadynamics and Electric Propulsion Laboratory, University of Michigan, 1919 Green Rd., Rm. B107, \\ Ann Arbor, Michigan 48109
}

(Received 2 February 1999; accepted 2 April 1999)

\begin{abstract}
When used in flowing electrostatically accelerated plasmas, electrostatic energy analyzers, such as Retarding Potential Analyzers (RPA's) or Cylindrical Mirror Analyzers (CMA's), occasionally yield data which seem implausible: for a known applied plasma acceleration voltage, electrostatic analyzers may indicate populations of ions having voltages much greater than that available through the electrostatic discharge. The process responsible for this phenomenon is resonant charge-exchange (CEX) collisions between ions of different charge species. Through the transfer of electrons, an ion of charge $n^{+}$can appear in an electrostatic analyzer with equivalent voltage of $n$-times the available acceleration potential. This paper discusses the phenomena responsible for the appearance of such high-voltage ions in the measured voltage distribution function and presents evidence for such reactions in the flowing xenon plasma produced by a Hall-effect current accelerator designed for spacecraft propulsion. For a $300 \mathrm{~V}$ applied potential on the Hall accelerator electrodes, CEX collisions are shown to produce ions having voltages as high as $800 \mathrm{~V}$ when measured using an electrostatic analyzer. (C) 1999 American Institute of Physics.
\end{abstract}

[S1070-664X(99)02307-1]

\section{INTRODUCTION}

Electrostatic energy analyzers, such as cylindrical mirror analyzers (CMA's) or gridded retarding potential analyzers (RPA's), enjoy widespread use in both laboratory and space plasma physics. Although geometries differ within this family of energy analyzers, the operation of such instruments relies on the use of a deflecting electrostatic field to alter the trajectory of plasma ions. Since the fields employed are purely electric, the ion trajectories within the analyzer are dependent not upon the ion energy, but rather upon the ratio of ion energy-per-charge, or equivalent acceleration voltage.

For a single charge-species plasma, the measured value of energy-per-charge for an ion of known charge can be straightforwardly manipulated to yield the true ion kinetic energy. However, many laboratory and space plasmas consist of at least trace amounts of ions having differing charge states. These multispecies flows can produce confusing results when subject to interrogation with an electrostatic analyzer technique. For example, the flowing xenon plasmas produced by Hall-effect current accelerators designed for spacecraft propulsion have traditionally been investigated with in situ gridded RPA probes to provide a measure of the ion acceleration voltage. ${ }^{1-3}$ The plasmas produced by Hall thrusters have been typically assumed to consist entirely of $\mathrm{Xe}^{+}$, and thus the resulting RPA data have been interpreted as a measurement of ion energy. Although this approximation seems warranted, as the plasma has been found to be almost $90 \% \mathrm{Xe}^{+}$, the remaining $10 \%\left(\mathrm{Xe}^{2+}\right.$ and $\left.\mathrm{Xe}^{3+}\right)$ cause

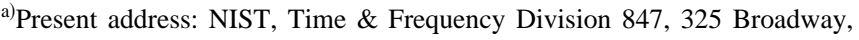
Boulder, Colorado 80303; electronic mail: lyon.king@boulder.nist.gov

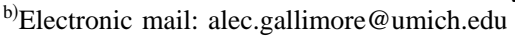

confusing results in the RPA data. Most notably, the existence of anomalous high-energy ions has been consistently documented with the RPA technique. ${ }^{1-3}$ Although a known acceleration voltage of $300 \mathrm{~V}$ was applied to the Hall thruster discharge, the RPA data indicated populations of ions having voltages as high as $500 \mathrm{~V}$. In the absence of a plausible explanation for such seemingly impossible highenergy ions, the RPA technique has recently been regarded as unreliable for use in Hall thruster plasmas.

Recent investigations have found that rather than arising from inherent shortcomings of the technique, the seemingly high-energy populations of ions found with the RPA are correct manifestations of various collision processes within the flowing plasma. One process capable of producing highenergy ions is the occurrence of momentum-transfer collisions within the multispecies flow. Due to the electrostatic acceleration within the thruster, the doubly charged $\mathrm{Xe}^{2+}$ ions acquire twice the kinetic energy of their singly charged counterparts, $\mathrm{Xe}^{+}$. Momentum-transfer collisions between the two species cause the $\mathrm{Xe}^{+}$ions to be "speeded up" by the overtaking $\mathrm{Xe}^{2+}$, which results in the growth of a long high-energy tail in the distribution function. Discussion of such momentum-transfer collisions will be the subject of a future paper. Another mechanism that can contribute to the population of seemingly high-energy ions is the chargeexchange (CEX) collision between different ionic species. In such collisions, the transfer of electrons from one species to another can cause electrostatic analyzer techniques to detect ions which appear to possess two, three, or even more times the kinetic energy available through the initial electrostatic acceleration process. The purpose of this paper is to discuss the phenomena responsible for the appearance of seemingly 
impossible high-energy populations of ions in electrostatic analyzer techniques arising from CEX collisions within a multispecies plasma. The properties of such CEX collisions are presented in terms of the measured ion energy-per-charge distribution function. Evidence of CEX collisions is then presented from data obtained in a flowing xenon plasma accelerated to $300 \mathrm{~V}$ by a Hall-effect ion beam thruster.

\section{COLLISION SIGNATURES}

The occurrence of CEX collisions within a multispecies ion beam can be predicted providing that the species densities as well as the cross sections of interest are known. Unfortunately, many cross sections for CEX collisions are not available within the scientific literature. Products of such collisions, however, leave distinct signatures in the measured ion-voltage distribution of electrostatically accelerated beams. These signatures have well-defined features which may be used to identify the reaction collision.

The sensitivity of electrostatic analyzers (such as CMA's or RPA's) to the quantity $E / q e$, where $E$ is the ion kinetic energy, $q$ is the integral charge state, and $e$ is the elementary charge, can be exploited to provide evidence of CEX collisions within the beam. For example, consider a two-species flow of identical mass ions, where one species has charge $q=1$ and the second has charge $q=2$. When accelerated through a potential $V$, both species will have identical $E / q e=V$, with the $q=2$ ion possessing twice the kinetic energy of the $q=1$ ion. A CEX collision is defined as an interaction during which one or more electrons are transferred between particles with no appreciable change in particle momentum. Thus, if the $q=1$ ion donates an electron to the $q=2$ ion, the resulting products will have the same energy as the reactants, however their charge state will be changed: the $q=2$ reactant will become a $q^{\prime}=1$ product (where primes denote post-CEX collision values), and likewise the $q=1$ reactant will become a $q^{\prime}=2$ product. The $q^{\prime}=1$ product, which was accelerated through $V$ with a precollision charge of $q=2$, will still have kinetic energy $E^{\prime}$ $=E=2 \mathrm{eV}$ and will therefore appear in the electrostatic analyzer as a product ion with voltage (energy-per-charge) $V^{\prime}$ $=2 \mathrm{~V}$. Similarly, the $q^{\prime}=2$ product was accelerated as a $q$ $=1$ ion and has energy of $E^{\prime}=E=1 \mathrm{eV}$, thus it will appear in the analyzer as an ion with energy-per-charge $V^{\prime}=V / 2$. Therefore, CEX collisions within a two-species beam composed of $q=1$ and $q=2$ ions accelerated through a potential of $V$ will produce product distributions at $V^{\prime}=2 \mathrm{~V}$ and $V^{\prime}$ $=V / 2$ when measured with an electrostatic energy analyzer.

Another property of the CEX process aids in identifying the products. Since CEX collisions involve negligible momentum transfer, such collisions preserve the shape of $f(V)$, the reactant voltage distribution function, within the product distributions. Consider, for example, the hypothetical distributions of $q=1$ and $q=2$ ions shown in Fig. 1. Such a distribution could be generated in an electric discharge plasma where doubly charged ions are created in the same region of space as the singly charged ions, albeit at a reduced rate, prior to acceleration through a potential $V$. CEX collisions between the two species conceptually involve a

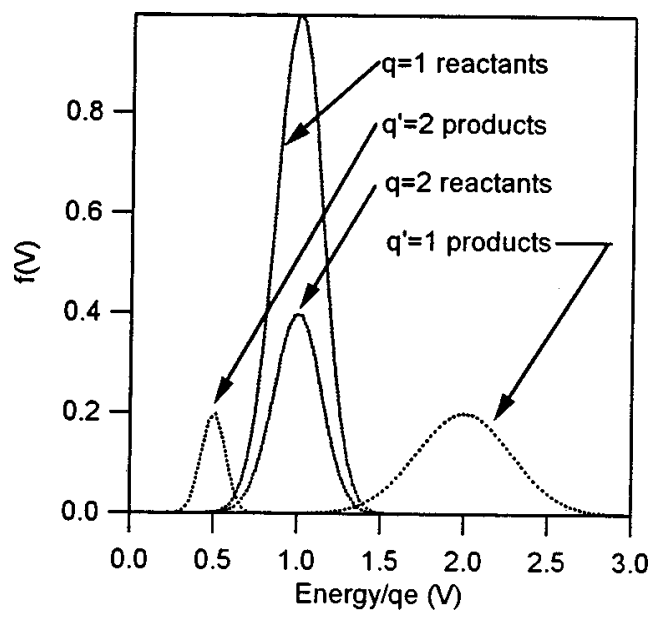

FIG. 1. Hypothetical ion-voltage distribution showing a Gaussian-like distribution of pre-CEX-collision reactants of $q=1$ and $q=2$ ions accelerated through an E/qe of $1 \mathrm{~V}$. Single electron transfer due to CEX can produce the product distributions shown with equivalent $E / q e$ of twice and one-half the accelerating potential.

"swap" of identities. Some fraction of the $q=1$ ions will give up an electron and become $q^{\prime}=2$ ions with no change of kinetic energy. If the CEX collision cross section is constant over the range of energies considered, the result will be a post-collision distribution of $q^{\prime}=2$ products having the same distribution shape as the $q=1$ reactants, however shifted to a voltage of $V^{\prime}=V / 2$. Concurrent with the voltage shift will be a narrowing of the distribution by a factor of $\frac{1}{2}$. Similarly, the $q=2$ reactant distribution will "give up" some ions to the CEX collision and result in a post-collision distribution of products with the same shape as the reactants, albeit shifted to a value of $V^{\prime}=2 \mathrm{~V}$ and broadened by a factor of 2.

The CEX product signatures can be determined in a straightforward fashion for flows consisting of more than two charge species. For example, reactant ions with $q=3$ and $q=1$ accelerated through potential $\mathrm{V}$ can produce CEX product distributions with voltages $V^{\prime}=V / 4, V / 3, V / 2,3 V / 4$, $3 V / 2$, and $3 V$, depending upon the number of electrons transferred during the collision and the direction of transfer. Concurrently, the product distributions will have the same shape as the reactant voltage distribution narrowed or broadened by the appropriate factor (i.e., the product distribution at $V^{\prime}=V / 4$ will have $\frac{1}{4}$ the width of the reactant distribution). With a known acceleration potential of $V$ applied to the ion beam, CEX collisions can produce ions having anomalous voltages of two or three times the available acceleration potential within the measured distribution. Such collision products are generally striking in the measured ion voltage signatures.

Another class of CEX collision which is relevant to the present discussion may occur between accelerated ions and macroscopically stagnant neutral particles. Such a collision will have a slightly different signature in the energy-percharge distribution. As an example, consider the singleelectron CEX collision between a $q=2$ ion accelerated through $V$ with a stagnant $q=0$ neutral. The products of this collision will be a distribution of $q^{\prime}=1$ located at $V^{\prime}=2 V$ 


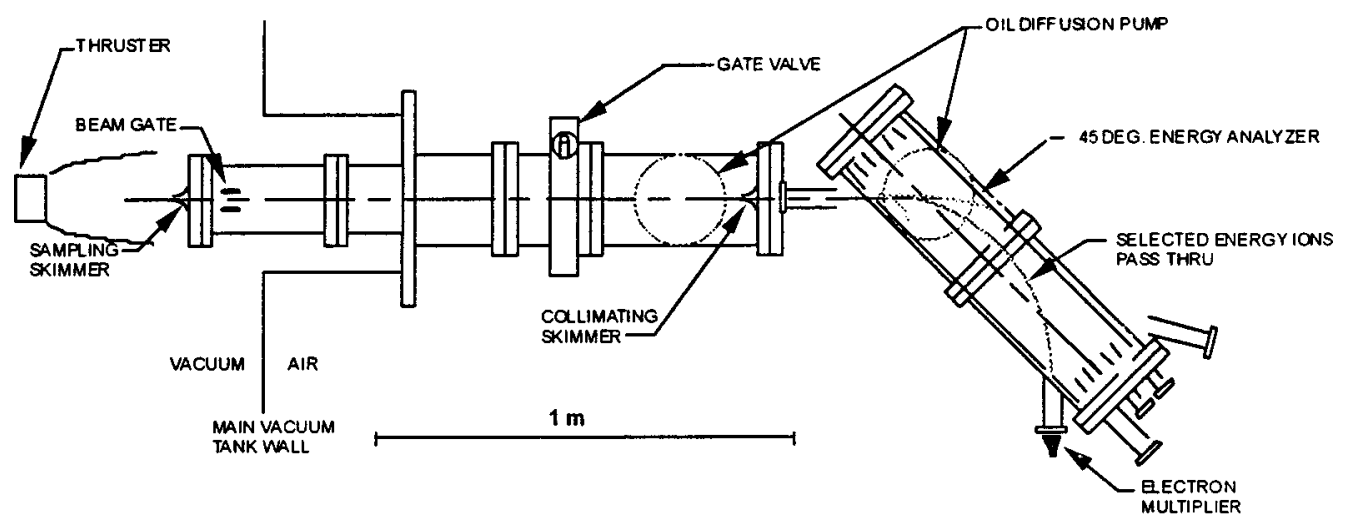

FIG. 2. Overall layout of MBMS system used to obtain ion-voltage distributions in flowing xenon plasma produced by Hall-effect accelerator. Schematic shows differentially pumped subchambers as well as $45-$ deg electrostatic energy analyzer.

having twice the width of the reactant $q=2$ population along with a $q^{\prime}=1$ distribution centered at $V^{\prime}=0$ with a width defined by the $q=0$ temperature.

\section{EXPERIMENTAL SETUP}

A search for CEX collision products was carried out in the flowing plasma plume of a Hall-effect current accelerator designed for space propulsion. This accelerator, a model SPT-100 manufactured by the Fakel Design Bureau of Russia, produced a 4.5-A beam of xenon ions accelerated through an applied potential of $300 \mathrm{~V}$. This operating point (current, voltage, and mass flow rate) was dictated by configuration of the dedicated power processing unit supplied with the thruster and optimized for space mission operation. The details of operation for the Hall accelerator in general, ${ }^{4-6}$ and the SPT-100 specifically, can be found in Refs. 1 and 7-9. The Hall accelerator was operated within a large space simulation vacuum facility at the University of Michigan. The facility consists of a 6-m-diameter by 9-mlong vacuum vessel evacuated by six $31-\mathrm{cm}$ oil diffusion pumps with a base pressure of $2.6 \mathrm{mPa}$. The Hall accelerator discharge was fed with $5 \mathrm{mg} / \mathrm{sec}$ of xenon for a steady-state working pressure of $6.6 \mathrm{mPa}$ within the chamber during operation.

The plasma plume was evaluated using a customdesigned Molecular Beam Mass Spectrometer (MBMS) integrated with the vacuum chamber. Although the MBMS was capable of providing mass species composition, the results of such diagnostics are omitted here and can be found in other references. ${ }^{10}$ For research reported in this paper, the MBMS was utilized as an electrostatic energy analyzer for the Hall accelerator plume ions in a species independent mode. A schematic of the MBMS can be found in Fig. 2. The MBMS system used a set of orifice skimmers to admit a beam of plume ions from the main vacuum chamber into an array of differentially pumped subchambers. The subchambers were maintained at high vacuum to minimize and effectively eliminate collisions involving ions within the beam. A sampling skimmer orifice was mounted on the upstream end of the MBMS, this orifice skimmed off a small diameter ion beam into the first subchamber. This beam was then colli- mated by a second orifice at the downstream end of the first subchamber. The collimated beam then passed through the entrance slit of a 45-deg electrostatic energy analyzer. This analyzer employed a constant electric field such that only ions with a preselected ratio of energy-per-charge had a trajectory which permits them to traverse the exit slit and impinge upon a detector. The Hall accelerator was mounted to a remotely controlled rotary table upstream of the inlet skimmer. By rotating the thruster relative to the fixed MBMS inlet, plasma diagnostics were completed as a function of angular position about the plume axis of symmetry. Since the plasma density was a maximum along the axis of symmetry, varying the thruster rotation angle allowed diagnostics to be performed within different density regimes. By repositioning the thruster mount between tests, data were obtained at both 0.5 and $1.0 \mathrm{~m}$ from the thruster exit plane.

The 45-deg electrostatic energy analyzer is a flexible, robust method for particle energy filtering that has been used widely in beam physics research. ${ }^{11-13}$ A schematic of the system utilized in the MBMS is shown in Fig. 3, with coordinate system and relevant dimensions defined. The ion beam is admitted through the entrance slit of the analyzer and immediately enters a region of constant electric field of magnitude $V_{p} / d$ oriented at an angle $\theta$ to the direction of travel. The ions thus experience a constant acceleration in the negative $y$-direction such that the spatial equation of their trajectory is

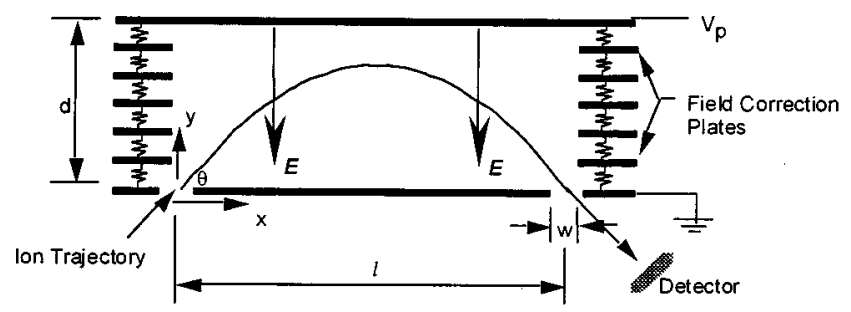

FIG. 3. Schematic of 45-deg electrostatic ion energy analyzer. Constant electric field is formed by applying repelling voltage $V_{p}$ to the top plate with the bottom plate grounded. Field correction plates are biased with a voltage divider to force boundary conditions at midplanes to prevent field distortion due to surrounding ground potential. 


$$
y=x-\frac{q_{i} e V_{p}}{2 d m_{i}} \frac{x^{2}}{u_{i}^{2} \sin ^{2} \theta},
$$

where $u_{i}$ is the ion velocity and $m_{i}$ is the ion mass. Since $\theta=45 \mathrm{deg}$ and $u_{i}^{2}=2 E_{i} / m_{i}$, Eq. (1) becomes

$$
y=x-\frac{1}{2 d} \frac{V_{p}}{\left(E_{i} / q_{i} e\right)} x^{2} .
$$

In order for an ion to pass through the analyzer and escape through the exit slit to the detector it must intersect the point $y=0, x=1$. This pass constraint is defined as the spectrometer constant, $K_{45}$, and is given by

$$
K_{45} \equiv \frac{V_{p}}{\left(E_{i} / q_{i} e\right)}=\frac{2 d}{1} .
$$

The analyzer thus performs the function of an energy-percharge filter, $E_{i} / q_{i} e$. Since the beam ions within the Hall accelerator plume experienced a discharge acceleration according to $q_{i} e V_{i}=1 / 2 m_{i} u_{i}^{2}$, the value of energy-per-charge for an ion is equivalent to the acceleration voltage, $V_{i}$, "felt" by the ion in the discharge acceleration chamber. For a given value of repelling plate voltage, only ions with

$$
V_{i}=\frac{1 / 2 m_{i} u_{i}^{2}}{q_{i} e}=\frac{V_{p}}{K_{45}}
$$

will reach the collector and be recorded as ion current. The current detector employed by the MBMS was a ceramic channel electron multiplier (CEM).

\section{RESULTS}

Ion-voltage distributions were obtained as a function of angle off plume axis for radial positions of 0.5 and $1.0 \mathrm{~m}$ from the Hall accelerator exit plane. These distributions were obtained by varying the allowed pass voltage of the 45-deg analyzer and measuring the resulting ion current on the CEM detector. In order to provide accurate values of ion voltage, an emissive probe was placed in the plasma $3 \mathrm{~cm}$ upstream of the MBMS inlet skimmer. The emissive probe provided a measurement of local plasma potential within the plume and outside of the sheath formed around the skimmer housing. Thus, the parasitic energy added to the ions as they "fell" from local plasma potential to the ground potential inside the MBMS could be subtracted from the data. The entire data set included traces of ion-voltage distribution for points along the complete 360-deg circumference about the thruster at a distance of $0.5 \mathrm{~m}$, with points along a total included arc of $260 \mathrm{deg}$ at $1.0 \mathrm{~m}$ from the thruster exit. The comprehensive data can be found in Ref. 10, with a representative subset included in this paper.

The major features of the ion-voltage distributions within $70 \mathrm{deg}$ of the plasma plume axis at $0.5 \mathrm{~m}$ are displayed by the traces of Fig. 4, which shows the measured curves for points lying at 10,30, 50, and $60 \mathrm{deg}$ off axis. These curves display a strong central "Gaussian-like" distribution centered at roughly $260 \mathrm{~V}$ which decays to small values for voltages greater than 300 and less than $200 \mathrm{~V}$. The ion-voltage distribution at $1.0-\mathrm{m}$ radius displayed significantly different features than comparable measurements at

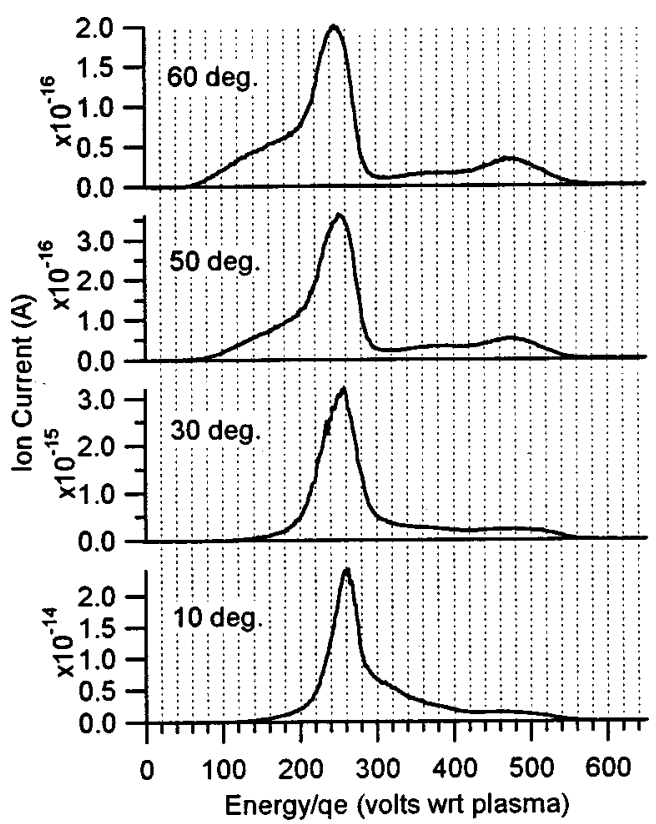

FIG. 4. Ion energy-per-charge distributions recorded at $0.5-\mathrm{m}$ radius from the Hall accelerator exit plane for angles of 10, 30, 50, and $60 \mathrm{deg}$ off the plasma plume axis of symmetry.

$0.5 \mathrm{~m}$. Figure 5 shows the measured voltage distribution for points at 10,30, 50, and $60 \mathrm{deg}$ off axis at $1.0 \mathrm{~m}$ from the thruster exit. Although the points at 30,50 , and 60 deg somewhat resemble their counterparts measured at $0.5 \mathrm{~m}$, the trace obtained at $10 \mathrm{deg}$ is strikingly different. In order to explore this difference more fully, Fig. 6 displays voltage distributions at finer angular resolution in the near-10-deg region of the plume at $1.0-\mathrm{m}$ radius.

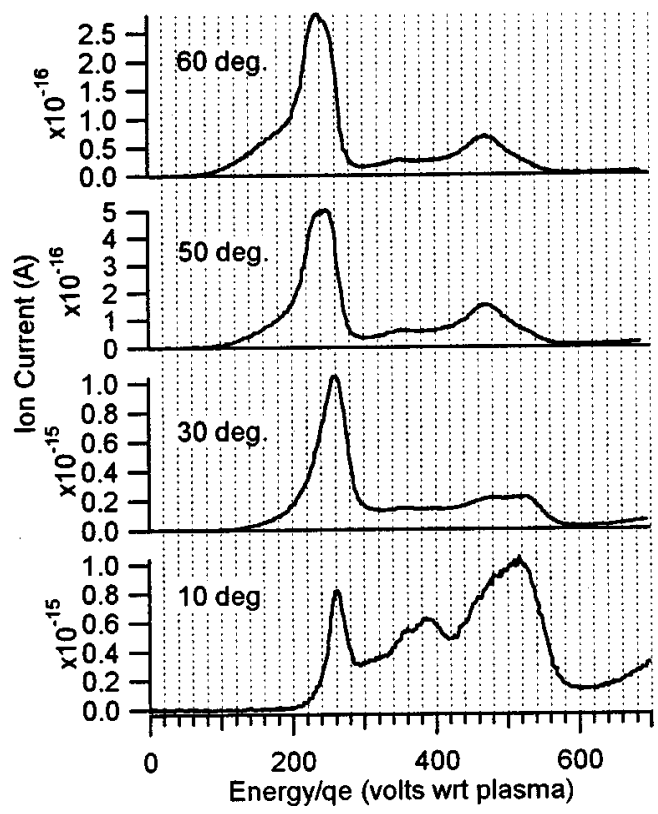

FIG. 5. Ion energy-per-charge distributions recorded at $1.0-\mathrm{m}$ radius from the Hall accelerator exit plane for angles of 10, 30, 50, and $60 \mathrm{deg}$ off the plasma plume axis of symmetry. 


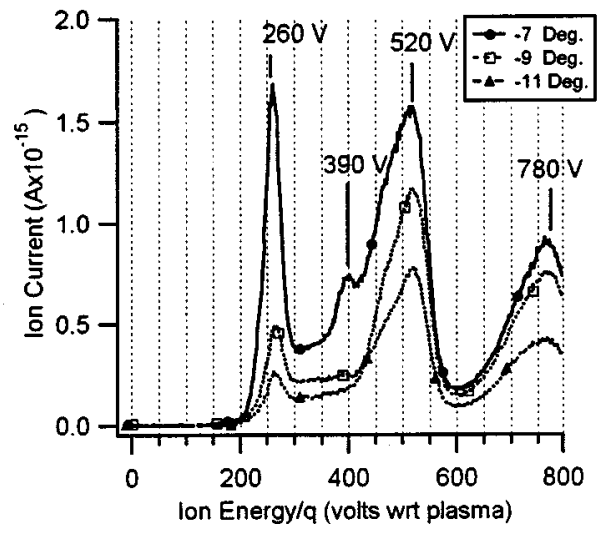

FIG. 6. Ion energy-per-charge distributions recorded at fine angular resolution in the vicinity of $10 \mathrm{deg}$ off plume axis at $1.0-\mathrm{m}$ radius from the thruster. Clearly present are distributions of ions having voltages much greater than the $300 \mathrm{~V}$ applied to the Hall accelerator electrodes.

\section{DISCUSSION}

The acceleration voltage applied to the Hall thruster was $300 \mathrm{~V}$ for the tests reported here. Thus, the tails of the ionvoltage distributions greater than $300 \mathrm{~V}$ could not have originated from this acceleration and a collisional explanation for their existence seems plausible. The long, monotonically decaying tails, such as displayed in the traces at 10 and $30 \mathrm{deg}$ in Fig. 4, are likely due to momentum transfer collisions between $q=2$ and $q=1$ ions. In such collisions, the more energetic $q=2$ ions yield some of their kinetic energy to the slower $q=1$ ions. Thus, the $q=1$ ions are speeded up and appear with energy-per-charge greater than $300 \mathrm{~V}$, while the $q=2$ ions which have lost energy to the collision appear as low-energy "tails." Discussion of such collisions is beyond the scope of this paper and can be found in Ref. 10. Of greater interest to this report is the "bump-on-tail" structure exhibited at voltages greater than $300 \mathrm{~V}$. It is noteworthy that the high-voltage tails obtained in the present study are of greater magnitude than those obtained with a similar RPA technique in an earlier investigation. ${ }^{3}$ The reason for this discrepancy is twofold: (1) the numerical differentiation required to obtain the ion-voltage distribution from the RPA data imposed an unavoidable noise signal on the data which made the magnitude uncertain for low ion current readings in the tail; (2) with a thruster discharge voltage of $300 \mathrm{~V}$ it is not intuitive to expect ions having much greater voltages, thus the earlier RPA investigation was limited in scope to voltages less than $500 \mathrm{~V}$ and the very high-voltage bumps were not discovered.

In order to identify the possible reactions accounting for the anomalous high-voltage ions, it is instructive to consider the Hall thruster plume flow as consisting of two interacting components: (1) high-voltage (on the order of 200-300 V) beam ions produced and accelerated within the thruster discharge chamber passing through (2) a background gas consisting of very low-energy macroscopically stagnant neutral particles due to either facility pumping limitations or unionized propellant. Possible reactions then include collisions between two beam ions and collisions between beam ions and low-energy neutrals. Through a mass spectrometric
TABLE I. Possible reactant and product combinations and the resulting product energy-per-charge (voltage) for CEX collisions occurring between two high-energy beam ions, each accelerated through voltage $V_{b}$ prior to collision.

\begin{tabular}{clc}
\hline \hline Reactants at $V_{b}$ & \multicolumn{1}{c}{ Ion products } & Electrons transferred \\
\hline $\mathrm{Xe}^{2+}+\mathrm{Xe}^{+}$ & $\mathrm{Xe}^{+}=2 V_{b}$ with $\mathrm{Xe}^{2+}=V_{b} / 2$ & 1 \\
$\mathrm{Xe}^{2+}+\mathrm{Xe}^{+}$ & $\mathrm{Xe}^{3+}=2 V_{b} / 3$ & 1 \\
$\mathrm{Xe}^{2+}+\mathrm{Xe}^{+}$ & $\mathrm{Xe}^{3+}=V_{b} / 3$ & 2 \\
$\mathrm{Xe}^{3+}+\mathrm{Xe}^{+}$ & $\mathrm{Xe}^{2+}=3 V_{b} / 2$ with $\mathrm{Xe}^{2+}=V_{b} / 2$ & 1 \\
$\mathrm{Xe}^{3+}+\mathrm{Xe}^{+}$ & $\mathrm{Xe}^{4+}=3 V_{b} / 4$ & 1 \\
$\mathrm{Xe}^{3+}+\mathrm{Xe}^{+}$ & $\mathrm{Xe}^{+}=3$ with $\mathrm{Xe}^{3+}=V_{b} / 3$ & 2 \\
$\mathrm{Xe}^{3+}+\mathrm{Xe}^{+}$ & $\mathrm{Xe}^{4+}=V_{b} / 4$ & 3 \\
$\mathrm{Xe}^{3+}+\mathrm{Xe}^{2+}$ & $\mathrm{Xe}^{2+}=3 V_{b} / 2$ with $\mathrm{Xe}^{3+}=2 V_{b} / 3$ & 1 \\
$\mathrm{Xe}^{3+}+\mathrm{Xe}^{2+}$ & $\mathrm{Xe}^{4+}=3 V_{b} / 4$ with $\mathrm{Xe}^{+}=2 V_{b}$ & 1 \\
$\mathrm{Xe}^{3+}+\mathrm{Xe}^{2+}$ & $\mathrm{Xe}^{+}=3 V_{b}$ with $\mathrm{Xe}^{4+}=V_{b} / 2$ & 2 \\
\hline \hline
\end{tabular}

study of the Hall thruster reported elsewhere, the plasma was found to consist mostly of $\mathrm{Xe}^{+}, \mathrm{Xe}^{2+}$, and $\mathrm{Xe}^{3+}$ ions in addition to parasitic neutral Xe. ${ }^{10}$ Considering all possible CEX reactions between $q=1,2$, and 3 beam ions yields the ion products as shown in Table I (considering only the formation of ions with $q<5$ ), with Table II tabulating the possible products of beam ion-neutral collisions. Since the MBMS can only detect ions with nonzero macroscopic energy, the possible products in Table II are limited to such ions.

The distributions at $0.5 \mathrm{~m}$ show a prominent "bump-ontail" residing at exactly twice the voltage of the main peak for the positions at 50, 60, and 90 deg off axis. For example, at $60 \mathrm{deg}$ the peak of the "main" beam distribution, $V_{b}$, occurs at $240 \mathrm{~V}$ with the peak in the "bump" distribution exactly at $2 V_{b}=480 \mathrm{~V}$. Similarly, the 30-, 50-, and 60-deg traces at $1.0 \mathrm{~m}$ display the same properties with the "bump" again occurring at $2 V_{b}$. Since the applied acceleration voltage on the Hall thruster was $300 \mathrm{~V}$, the ions possessing voltages much greater than $300 \mathrm{~V}$ must have arisen from collisions within the plasma. From Tables I and II, the possible reactions creating products with a voltage of twice the main beam voltage are (1) a CEX collision between a $\mathrm{Xe}^{+}$beam ion and $\mathrm{Xe}^{2+}$ beam ion, (2) the CEX collision between a $\mathrm{Xe}^{3+}$ beam ion and a $\mathrm{Xe}^{2+}$ beam ion, and (3) the CEX collision between a $\mathrm{Xe}^{2+}$ beam ion and a background neutral.

If the reaction responsible for creation of the peak at $2 V_{b}$ is of the first type mentioned above, between $q=1$ and $q$ $=2$ beam ions, then there must also exist a sibling distribution centered at $V_{b} / 2$. The data do not indicate such a lowvoltage sibling peak. Similarly, the second reaction spawns a peak at $3 V_{b} / 4$ which is also absent. It then seems apparent

TABLE II. Possible reactant-product combinations for the CEX collision between a beam ion accelerated through voltage $V_{b}$ prior to collision with a macroscopically stagnant background neutral.

\begin{tabular}{clc}
\hline \hline $\begin{array}{c}\text { Reactant ion at } V_{b} \\
\text { with } 0 \mathrm{~V} \text { neutral }\end{array}$ & $\begin{array}{c}\text { Ion products with } \\
\text { energy }>0\end{array}$ & Electrons transferred \\
\hline $\mathrm{Xe}^{+}+\mathrm{Xe}$ & none & N/A \\
$\mathrm{Xe}^{2+}+\mathrm{Xe}$ & $\mathrm{Xe}^{+}=2 V_{b}$ & 1 \\
$\mathrm{Xe}^{3+}+\mathrm{Xe}$ & $\mathrm{Xe}^{2+}=3 V_{b} / 2$ & 1 \\
$\mathrm{Xe}^{3+}+\mathrm{Xe}$ & $\mathrm{Xe}^{+}=3 V_{b}$ & 2 \\
\hline \hline
\end{tabular}


that the single-electron CEX collision between fast $\mathrm{Xe}^{2+}$ and neutral Xe accounts for the high-voltage peak in the distribution seen at roughly twice the discharge acceleration voltage. A statistical collision analysis of the plasma supports this finding. ${ }^{10}$ Although the CEX cross sections for multiply charged $\mathrm{Xe}^{n+}(n>1)$ collisions with neutral $\mathrm{Xe}$ are not available for the energy range of interest to this study, the cross section for $\mathrm{Xe}^{+}$with $\mathrm{Xe}$ can be computed according to the method of Rapp and Francis. ${ }^{14}$ For the background pressure present in this test of $6 \mathrm{mPa}$ and an ion velocity corresponding to an acceleration of $300 \mathrm{~V}$, the mean-free path for $\mathrm{Xe}^{+}+\mathrm{Xe}$ charge exchange is approximately $1.5 \mathrm{~m}$. This equates to a $30 \%$ probability of a CEX collision over a path length of $0.5 \mathrm{~m}$. Since the CEX cross section scales with $q^{1.3}$ for xenon, ${ }^{15}$ the collision probabilities for $\mathrm{Xe}^{n+}$, where $n>1$, will be greater than $30 \%$.

Since the dominant CEX collisions observed in this study occur between fast beam ions and ambient background neutrals, one should expect to see more CEX products with increasing ion path length through the neutrals. Indeed, examination of Fig. 5 showing data at $1.0 \mathrm{~m}$ indicates that the CEX distribution existing at $2 V_{b}$ is of larger magnitude than for corresponding angular positions recorded at $0.5 \mathrm{~m}$ from the thruster exit. As an example, consider the traces taken at $50 \mathrm{deg}$ off axis for distances of 0.5 and $1.0 \mathrm{~m}$ from the thruster. Since the available acceleration voltage was limited to $300 \mathrm{~V}$, all ions detected with apparent voltages greater than $300 \mathrm{~V}$ must be the products of CEX collisions, while those lying below $300 \mathrm{~V}$ represent ions which have not undergone a CEX collision. Numerically integrating the measured voltage distributions at $50 \mathrm{deg}$ to compute the total number of CEX product ions having voltages greater than $300 \mathrm{~V}$ reveals that $20 \%$ of the total ion population at $0.5 \mathrm{~m}$ are collision products, while such products comprise $40 \%$ of the population at $1.0 \mathrm{~m}$.

The effect of ion path length on collision probability is most apparent for the traces near the center line taken at a distance of $1.0 \mathrm{~m}$ from the thruster. The data of Fig. 6 display very prominent high-voltage peaks which have locations corresponding to all of the possible post-collision voltage multiples listed in Table II. For example, the main beam distribution which was produced through thruster acceleration is centered at $V_{b}=260 \mathrm{~V}$ and extends from 220 to about $300 \mathrm{~V}$. The CEX product peak attributed to single-electron transfer to $\mathrm{Xe}^{2+}$ from a neutral atom lies exactly at $2 V_{b}$ $=520 \mathrm{~V}$ and has roughly twice the width of the main distribution. Similarly, the distribution associated with a twoelectron transfer to $\mathrm{Xe}^{3+}$ lies precisely at $3 V_{b}=780 \mathrm{~V}$ and has three times the width of the primary beam population, while the distribution from single-electron transfer to $\mathrm{Xe}^{3+}$ can be found at $3 V_{b} / 2=390 \mathrm{~V}$. From these data it is apparent that the high-voltage product distributions have magnitudes comparable to or greater than the primary thruster-produced distribution. This indicates that on the order of $50 \%$ of the effluent plasma suffers a CEX collision within the $1.0 \mathrm{~m}$ path from the thruster. Based on available cross sections, such high collision probability is to be expected for the high plasma-density region near the plume axis at $1.0 \mathrm{~m}$ from the thruster.

Additional support for the prevalence of CEX collisions was presented previously in Ref. 3. This study used a neutral particle flux probe to investigate the flux of energetic neutral atoms in the plasma. The results indicated that a large population of highly energetic neutrals existed within 20 deg of the thrust axis. These neutrals were identified as the products of single-electron CEX between accelerated $\mathrm{Xe}^{+}$and stagnant background Xe, producing an energetic post-collision $\mathrm{Xe}$ together with a stagnant population of $\mathrm{Xe}^{+}$.

\section{ACKNOWLEDGMENTS}

This research benefited from the generous support of the Air Force Office of Scientific Research represented by Dr. Mitat Birkan, the NASA-Lewis Research Center, with equipment grants administered by Mr. John Sankovic, and support from the NASA-Johnson Space Center under the direction of Mr. Richard Barton. The unique opportunity to evaluate a state-of-the-art Hall thruster was made available by a generous equipment loan from Mr. Michael Day of the Space Systems/Loral company. This support is gratefully acknowledged. Additionally, the authors would like to thank technicians Warren Eaton, Terry Larrow, Gary Gould, Tom Griffin, and Dave McLean for assistance with hardware fabrication. The first author would also like to thank the research staff of PEPL namely Matt Domonkos, Colleen Marrese, Frank Gulczinski, James Haas, Sang-wook Kim, and George Williams for their discussions in the preparation of this manuscript.

${ }^{1}$ S. Absalamov, V. Andreev, T. Colbert, M. Day, V. Egorov, R. Gnizdor, H. Kaurman, V. Kim, A. Korakin, K. Kozubsky, S. Kudravzev, U. Lebedev, G. Popov, and V. Zhurin, Measurement of Plasma Parameters in the Stationary Plasma Thruster (SPT-100) Plume and its Effect on Spacecraft Components, AIAA-92-3156, Proceedings of the 28th AIAA/SAE/ASME/ ASEE Joint Propulsion Conference, Nashville, TN, 1992 (American Institute of Aeronautics and Astronautics, Washington DC, 1992).

${ }^{2}$ R. Myers and D. Manzella, Stationary Plasma Thruster Plume Characteristics, IEPC-93-096, Proceedings of the 23rd International Electric Propulsion Conference, Seattle, WA, 1993 (Electric Rocket Propulsion Society, Worthington, OH, 1993).

${ }^{3}$ L. B. King, A. D. Gallimore, and C. M. Marrese, J. Propul. Power 14(3), 327-335 (1998).

${ }^{4}$ H. Kaufman, AIAA J. 23(1), 78-87 (1985).

${ }^{5}$ V. Zharinov and Y. Popov, Sov. Phys. Tech. Phys. 12, 208-211 (1967).

${ }^{6}$ A. Bishaev and V. Kim, Sov. Phys. Tech. Phys. 23, 1055-1057 (1978).

${ }^{7}$ J. R. Brophy, J. W. Barnett, J. M. Sankovic, and D. A. Barnhart, Performance of the Stationary Plasma Thruster: SPT-100, Proceedings of 28th AIAA/SAE/ASME/ASEE Joint Propulsion Conference, Nashville, TN, 1992 (American Institute of Aeronautics and Astronautics, Washington DC, 1992).

${ }^{8}$ J. Sankovic, J. Hamley, and T. Haag, Performance Evaluation of the Russian SPT-100 Thruster at NASA LeRC, IEPC-93-094, Proceedings of 23rd International Electric Propulsion Conference, Seattle, WA, 1993 (Electric Rocket Propulsion Society, Worthington, OH, 1993).

${ }^{9}$ C. E. Garner, J. E. Polk, K. D. Goodfellow, and J. R. Brophy, Performance Evaluation and Life Testing of the SPT-100, IEPC-93-091, Proceedings of 23rd International Electric Propulsion Conference, Seattle, WA, 1993 (Electric Rocket Propulsion Society, Worthington, OH, 1993).

${ }^{10}$ L. B. King, "Transport-property and mass spectral measurements in the plasma exhaust plume of a Hall-effect space propulsion system,' Doctoral 
Dissertation, Dept. of Aerospace Engineering, University of Michigan, 1998.

${ }^{11}$ W. deZeeuw, H. van der Ven, J. de Wit, and J. Donne, Rev. Sci. Instrum. 62(1), 110-117 (1991).

${ }^{12}$ A. Gaus, W. Htwe, T. Brand, and M. Schulz, Rev. Sci. Instrum. 65(12), 3739-3745 (1994).
${ }^{13}$ V. Esaulov, O. Grizzi, L. Guillemot, M. Huels, S. Lacombe, and Vu Ngoc Tuan, Rev. Sci. Instrum. 67(1), 135-144 (1996).

${ }^{14}$ D. Rapp and W. Francis, J. Chem. Phys. 37(11), 2631-2645 (1962).

${ }^{15}$ T. Kusakabe, T. Horuchi, N. Nagai, H. Hanaki, I. Konomi, and M. Sakisaka, J. Phys. B 19, 2165-2174 (1986). 